\title{
Токовый шум и падение эффективности светодиодов при туннелировании носителей из квантовой ямы InGaN/GaN с участием дефектов
}

\author{
(ㄱ Н.И. Бочкарева, А.М. Иванов, А.В. Клочков, Ю.Г. Шретер \\ Физико-технический институт им. А.Ф. Иоффре Российской академии наук \\ 194021 Санкт-Петербург, Россия \\ ๑ E-mail: y.shreter@mail.ioffe.ru
}

(Получена 29 мая 2018 г. Принята к печати 13 июня 2018 г.)

\begin{abstract}
Проведены измерения токовых зависимостей спектральной плотности токового шума и квантовой эффективности в зеленых и синих светодиодах с квантовыми ямами InGaN/GaN. Показано, что уровень шума сильно растет в области больших токов, при которых наблюдается падение квантовой эффективности. Механизм формирования токового шума связывается с прыжковым транспортом по состояниям центров окраски в $\mathrm{GaN}$ через $n$-барьер квантовой ямы InGaN/GaN. Источником шума является прыжковое сопротивление области пространственного заряда, ограничивающее ток термоактивированных электронов в квантовую яму. Падение эффективности и рост уровня шума связываются с изменением направления электрического поля вблизи квантовой ямы при высоких уровнях инжекции и увеличением туннельной утечки дырок из квантовой ямы. Показано, что экспериментальные частотные спектры токового шума, имеющие при рабочих токах вид лоренцевского спектра, связаны с частотой перескока между глубокими центрами вблизи квантовой ямы $\mathrm{InGaN} / \mathrm{GaN}$ и максвелловской релаксацией в область пространственного заряда.
\end{abstract}

DOI: 10.21883/FTP.2019.01.46996.8847

\section{1. Введение}

Уровень низкочастотных флуктуаций тока (токового шума) в электронных и оптоэлектронных приборах на основе $\mathrm{GaN}$, материалах с высокой плотностью протяженных и точечных дефектов, является важнейшим параметром, часто имеющим решающее значение для общей работоспособности прибора. В НЕМТ-транзистоpax шум тока утечки в $\mathrm{AlGaN} / \mathrm{GaN}$ барьерах Шоттки, используемых в качестве затвора, вызывает нестабильность характеристик транзистора и значительные шумы тока канала $[1,2]$. В $\mathrm{GaN}$-светодиодах токовые шумы являются чувствительным индикатором надежности прибора, деградационных процессов и длительности срока службы [3-7].

В литературе представлены различные точки зрения на физическую природу и механизм формирования токового шума в барьерных структурах на основе GaN. Ocновной компонентой шума считается шум $1 / f$ (фликкершум), но в литературе нет единой точки зрения на механизм возникновения шума $1 / f$ и его источники. В НЕМТ-транзисторах с треугольной квантовой ямой на гетерогранице $\mathrm{AlGaN} / \mathrm{GaN} 1 / f$-шум тока канала связывается с флуктуациями занятости центров, формирующих хвосты плотности состояний у краев зон в $\mathrm{GaN}$ [8], подобно $1 / f$-шуму объемного $\mathrm{Si}$ и $\mathrm{GaAs}[9]$; туннелированием из двумерного электронного газа в глубокие ловушки или состояния хвостов плотности состояний в слоях $\mathrm{GaN}$ и $\mathrm{AlGaN} \mathrm{[10];} \mathrm{флуктуациями} \mathrm{подвижности}$ при рассеянии на заряженных дислокациях [11].

В светодиодных структурах с квантовой ямой $\mathrm{AlGaN} / \mathrm{InGaN} / \mathrm{GaN}$ появление ступеней на экспериментальных токовых и частотных зависимостях плотности шума часто связывается с суперпозицией двух или трех независимых шумовых компонент: шума $1 / f$ $p$-n-перехода [3] и шума $1 / f$ последовательного сопротивления диода (шума базы и/или контактного шума) $[3,12]$, генерационно-рекомбинационного шума [12], а также случайного телеграфного шума [3]. В то же время исследования избыточного шума синих светодиодов на основе $\mathrm{GaN}$ показали, что основным источником токового шума является прыжковый транспорт по состояниям дефектов в области пространственного заряда (ОПЗ) барьеров квантовой ямы AlGaN/InGaN/GaN [13]. Эти исследования показали также, что характер связи между уровнем токового шума и величиной квантовой эффективности светодиода не всегда однозначен $[13,14]$. В то же время падение эффективности с ростом плотности тока, характерное для InGaN/GaN светодиодов, сопровождается сильным ростом плотности токового шума $[13,14]$. Это указывает на общую причину этих процессов. В работе [15] показана связь эффекта падения эффективности в синих светодиодах с прыжковым транспортом через ОПЗ по глубоким состояниям центров окраски в $\mathrm{GaN}$, ответственных за полосы фотолюминесценции. Это может позволить не только понять источник шума, но и определить тип дефектов, ответственных за шум и падение эффективности. Эффективность падает в результате ослабления конфайнмента носителей в квантовой яме и их безызлучательной рекомбинации в барьерах ямы [16]. Характерное время перескока между локализованными центрами в ОПЗ с широким энергетическим спектром дефектов может меняться на несколько порядков величины, поэтому представляет интерес сопоставить токовые зависимости эффективности и уровня токового шума на различных частотах анализа. Такие исследования помогут также установить причины низкой эффективности зеленых светодиодов на основе 
$\mathrm{GaN}$, еe уменьшения при увеличении концентрации индия в квантовой яме InGaN/GaN [17] и найти подход к решению этой проблемы.

С этой целью в работе изучаются особенности поведения тока, квантовой эффективности, плотности токового шума при увеличении прямого смещения и сканировании квазиуровнями Ферми энергетического распределения глубоких центров в $p-n$-структурах зеленых и синих светодиодов. В разд. 2 дается описание методики измерения токового шума на различных частотах анализа. В разд. 3 сопоставляются экспериментальные токовые зависимости плотности токового шума и эффективности, а также зависимости плотности шума и фактора идеальности ВАХ от прямого смещения, позволяющие сделать вывод о доминирующем влиянии прыжкового транспорта в $p-n$-структурах в квантовой ямой $\mathrm{InGaN} / \mathrm{GaN}$ на токовый шум. В разд. 4 обсуждается модель, описывающая механизм формирования токового шума при туннелировании носителей через ОПЗ с участием донорноакцепторных комплексов, являющихся центрами окраски в $\mathrm{GaN}$. Как будет показано далее, источником избыточного токового шума является прыжковое сопротивление ОПЗ, а частотный спектр шума определяется частотой перескока между центрами и временем максвелловской релаксации в ОПЗ. С ростом прямого смещения вблизи квантовой ямы происходит сначала спрямление зон, а затем изменение знака изгиба зон, что приводит к туннелированию дырок из квантовой ямы и их последующей безызлучательной рекомбинации в $n$-барьере, вызывающих падение эффективности и рост уровня шума.

\section{2. Эксперимент}

В работе представлены результаты, полученные на коммерческих планарных светодиодах Nichia с одиночной квантовой ямой $p-\mathrm{AlGaN} / \mathrm{In}_{x} \mathrm{Ga}_{1-x} \mathrm{~N} / n-\mathrm{GaN}$ толщиной $30 \AA[18,19]$, пиковой энергией излучения $h v_{\mathrm{p}}=2.34$ и 2.46 эВ (зеленые диоды А и В соответственно) и $h v_{\mathrm{p}}=2.7$ эВ (синие диоды С). Рабочий ток диодов $20 \mathrm{MA}$, реальная площадь $\sim 10^{-3} \mathrm{~cm}^{2}$. Результаты измерений и детальный анализ вольт-амперных характеристик, зависимостей от прямого напряжения внешней квантовой эффективности и дифференциальной емкости на частоте 1 МГц, светодиодов А, В и С представлены в работе [16].

Измерения плотности токового шума были проведены в полосе частот 10 Гц-7.3 кГц. Измерялись флуктуации тока $\delta I_{\text {meas }}$, возникающие при пропускании прямого тока через светодиод. Флуктуации напряжения на нагрузочном сопротивлении $R_{\mathrm{L}}=100$ Ом оцифровывались измерительным аналого-цифровым преобразователем с собственным уровнем шумов 1 мкВ в полосе частот 7.3 кГц. При измерениях записывались 2 млн выборок с частотой дискретизации 16 кГц. Далее осуществлялась обработка данных программой с использованием быстрого преобразования Фурье. Шум рассчитывался в четырех полосах равной ширины - 17.6 Гц с центральными частотами $f=20,70,270$ и 1000 Гц. Затем рассчитывалась спектральная плотность шума $S_{I}$. Флуктуации тока короткого замыкания вычислялись как $\delta I=\delta I_{\text {meas }}\left(1+R_{\mathrm{L}} / r_{d d}\right)$, где $r_{d d}$ - дифференциальное сопротивление светодиода.

\section{3. Результаты эксперимента}

На рис. 1, $a-c$ представлены результаты измерений токовых зависимостей плотности токового шума $S_{I}(I)$ для частот анализа $f=20,70,270,1000$ Гц (кривые 1-4) и квантовой эффективности (кривые 5) светодиодов А-С. Как видно из рис. 1, между уровнем шума и эффективностью светодиода нет однозначной корреляции. Так, у „синего“ диода С, эффективность которого принята на единицу (рис. 1, $c$ ), и у „зеленого“ светодиода В с эффективностью, в 2 раза меньшей (рис. 1,b), плотности токового шума в области рабочих токов отличаются незначительно, тогда как в области малых токов $I=0.1 \mathrm{мA} \mathrm{уровень} \mathrm{шума} \mathrm{диода} \mathrm{В} \mathrm{на} \mathrm{порядок} \mathrm{выше.}$ В то же время у „зеленого“ диода А с эффективностью, в 2 раза меньшей, чем у диода $\mathrm{B}$, при рабочем токе 20 мА плотность токового шума выше почти на порядок, но в области малых токов уровень шума близок к уровню шума „синего“ диода С. У всех диодов плотность токового шума растет с понижением частоты анализа, но характер зависимостей $S_{I}(I)$ существенно различен. Основной рост плотности токового шума наблюдается при токах, превышающих пиковый ток $I_{\mathrm{p}}$, при котором наблюдается максимум эффективности. Это позволяет предположить, что причина падения эффективности и роста уровня шума связана с особенностями механизма тока при напряжениях выше пикового напряжения $V_{j \mathrm{p}}$.

На рис. 2, $a-c$ зависимости плотности токового шума $S_{I}(I)$ в области частот $20,70,270,1000$ Гц (кривые 1-4) и квантовой эффективности (кривые 6) светодиодов $\mathrm{A}-\mathrm{C}$ представлены как функции напряжения на $p$-n-переходе $V_{j}$, определенного как $V_{j}=V-I r_{s}$. Здесь $V$ - приложенное к диоду напряжение, $r_{s}$ - последовательное сопротивление. Последовательное сопротивление оценивалось из наклона $I-V$-характеристик, и при рабочих токах составило в диодах А, В и $\mathrm{C} r_{s}=20,11.5$ и 26 Ом соответственно. Кривые $\log I\left(V_{j}\right)$, представленные на вставке к рис. 2, $b$, могут быть аппроксимированы экспоненциальной функцией $I \propto \exp \left(q V_{j} / n_{j} k T\right)$, где $n_{j}\left(V_{j}\right)$ - фактор идеальности, $k T-$ тепловая энергия, $q$ - элементарный заряд. Зависимости фактора идеальности $I-V_{j}$-характеристик $n_{j}\left(V_{j}\right)=(q / k T) d V_{j} / d \ln I$ от напряжения $V_{j}$ представлены на рис. 2, $a-c$ (кривые 5). Из рис. 2 видно, что в области малых напряжений крутизна BAX наибольшая у „синего“ светодиода С, при этом фактор идеальности уменьшается до $n_{j}=2$, величины, характерной для $p-n$-структуры с надбарьерной инжекцией, для которой $2 \geq n_{j} \geq 1$. У ,зеленых“ светодиодов А и $\mathrm{B} n_{j}>2\left(n_{j} \approx 2.5\right.$ и 6 соответственно). В области напряжений $V_{j}=2.6 \mathrm{~B}$ крутизна ВАХ становится примерно одинаковой у всех диодов $\left(n_{j} \approx 3\right)$, как и величина тока $(I=45-60$ мкА). При дальнейшем увеличении напряжения $V_{j}>2.6 \mathrm{~B}$ у диодов наблю- 

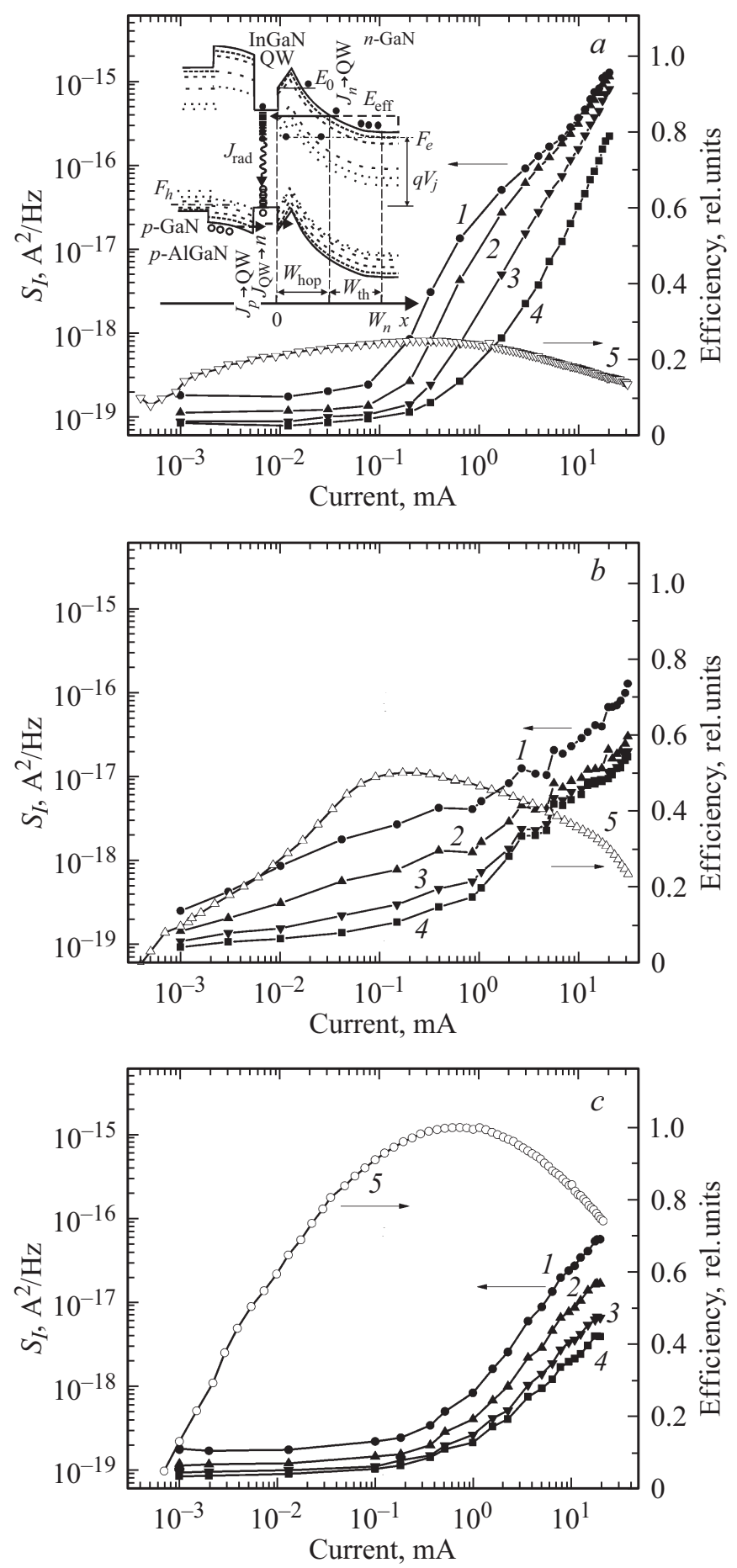

Рис. 1. Зависимости спектральной плотности токового шума $(1-4)$ и эффективности $(5)$ от прямого тока для различных частот анализа в светодиодах: $a-\mathrm{A}, b-\mathrm{B}$ и $c-\mathrm{C}$. Частота анализа, $f$, Гц: $1-20,2-70,3-270,4-1000$. На вставке к рис. $1, a-$ схематическое изображение зонной структуры $\mathrm{GaN}$-светодиода с одиночной квантовой ямой $\mathrm{InGaN} / \mathrm{GaN}$ при прямом смещении. Пунктирными линиями отмечены максимумы плотности состояний для центров окраски, ответственных за полосы дефектной фотолюминесценции. $J_{n} \rightarrow \mathrm{QW}$ и $J_{p} \rightarrow \mathrm{QW}-$ электронная и дырочная компоненты прыжкового тока через ОПЗ $n$-барьера квантовой ямы, $E_{\text {eff }}-$ транспортная энергия для электронов, формирующих пик в спектре излучения.
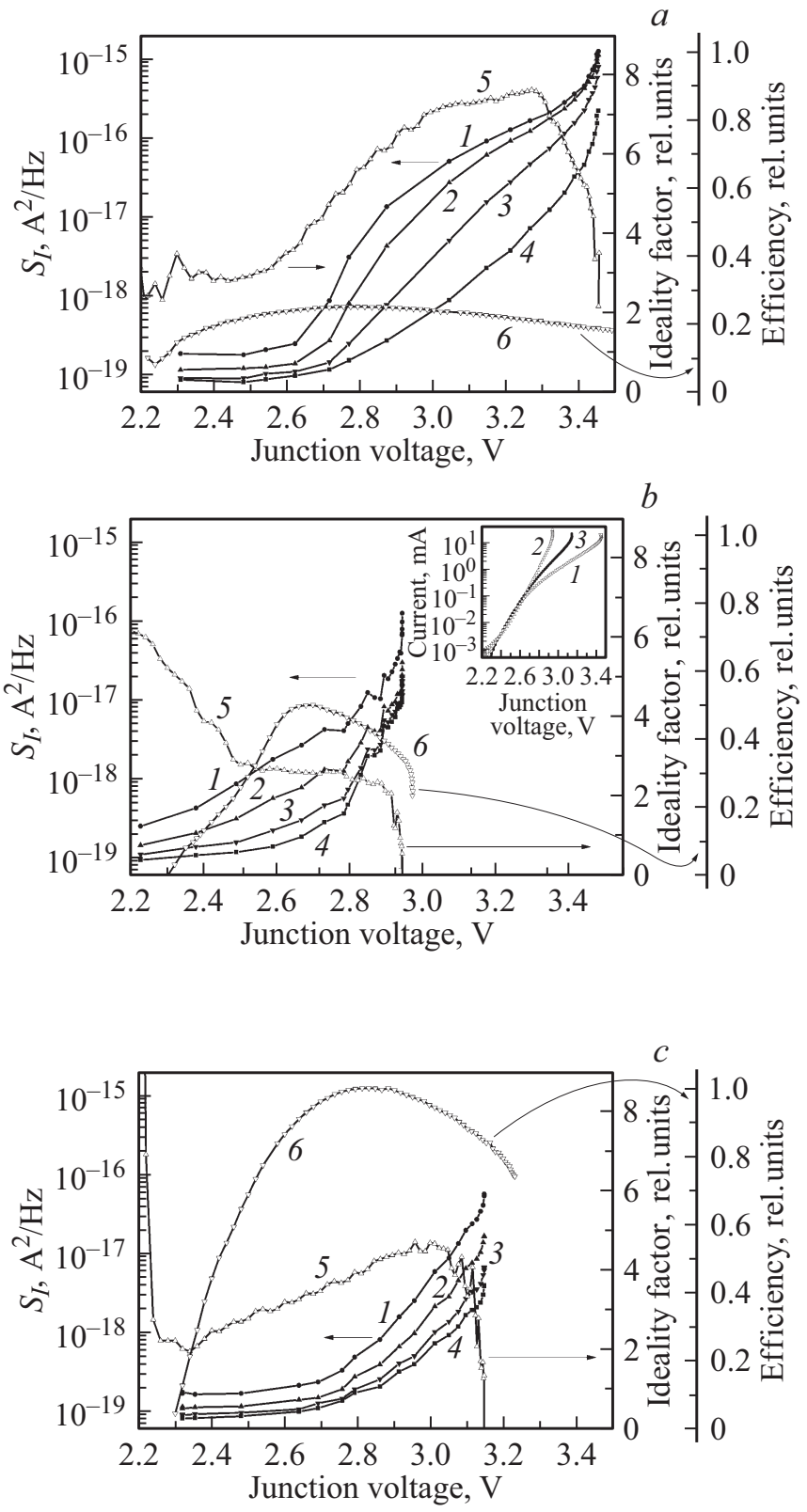

Рис. 2. Зависимости спектральной плотности токового шума $(1-4)$, эффективности (5) и фактора идеальности $I-V_{j}$-характеристик (6) от напряжения на $p-n$-переходе для различных частот анализа в светодиодах: $a-\mathrm{A}, b-\mathrm{B}$ и $c-$ С. Частота анализа, $f$, Гц: $1-20,2-70,3-270$, $4-1000$. На вставке к рис. $2, b-I-V_{j}-$ характеристики светодиодов А (1), В (2) и С (3).

дается существенное различие в величине крутизны BAX. У диода В с ростом напряжения крутизна ВАX увеличивается и $n_{j}$ уменьшается, у диода А крутизна $\mathrm{BAX}$ уменьшается и $n_{j}$ увеличивается до $n_{j} \approx 7.5$, а у диода $\mathrm{C}$ до $n_{j} \approx 4.5$. Крутизна $\mathrm{BAX}$ у диодов $\mathrm{A}$ и $\mathrm{C}$ вновь увеличивается при $V_{j}=3.3$ и 3 В соответственно.

Характер роста уровня шума с напряжением обнаруживает связь с изменением крутизны ВАХ. В области малых напряжений уровень шума на низких частотах 


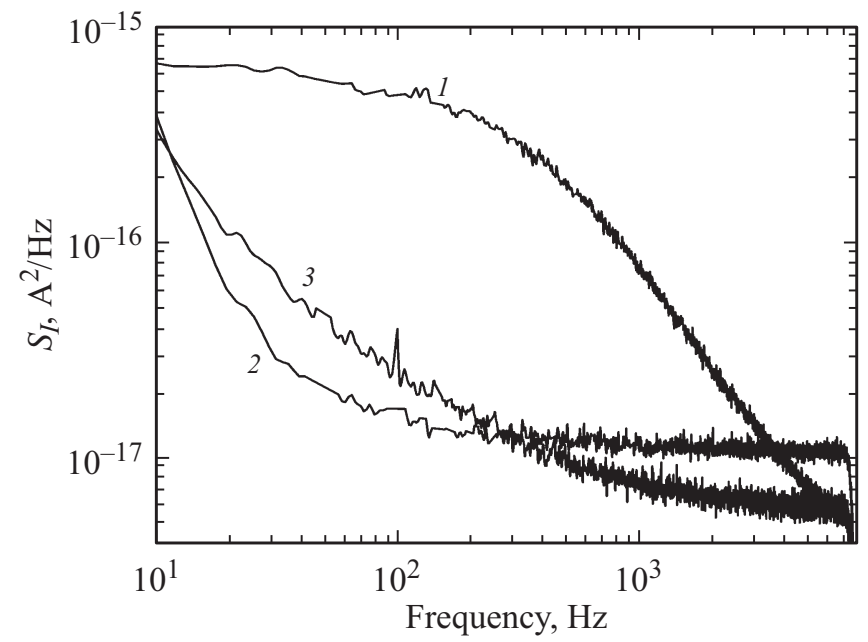

Рис. 3. Частотные зависимости спектральной плотности токового шума в светодиодах А (1), В (2) и С (3). Ток, мА: $1,2,3-20$.

растет с $V_{j}$ наиболее сильно у диода В с высоким фактором идеальности, равным $n_{j}=6$ при $V_{j}=2.2 \mathrm{~B}$ (рис. 2,b). В области средних напряжений уровень низкочастотного шума у диода А с наибольшим фактором идеальности и низкой эффективностью растет с напряжением в соответствии с ростом фактора идеальности $n_{j}\left(V_{j}\right)$ (рис. 2,a). В области рабочих напряжений у всех диодов падение фактора идеальности с ростом $V_{j}$ сопровождается сильным ростом уровня шума (рис. $2, a-c$ ).

На рис. 3 представлены частотные спектры плотности токового шума $S_{J}(f)$ исследованных светодиодов, измеренные при рабочем токе 20 мА. В частотном диапазоне 10 Гц $<f<7.3$ кГц спектр „зеленого“ диода А имеет форму, близкую к форме лоренцовского спектра: в области низких частот $10-100 \Gamma_{ц} S_{I}(f)$ слабо уменьшается с повышением частоты, а в области $f>200$ Гц характер уменьшения $S_{I}$ с частотой близок к зависимости $S_{I} \sim 1 / f^{2}$. Зависимость $S_{I}(f)$,зеленого“ диода В близка к зависимости $S_{I} \sim 1 / f^{2}$ лишь в области низких частот $f<40$ Гц. В „синем“ диоде С спектр $S_{I}(f)$ имеет форму, близкую к спектру $1 / f$ шума: $S_{I} \propto 1 / f$. Однако в области частот $10-100$ Гц наблюдается более сильный рост шума с понижением частоты $S_{I} \propto 1 / f^{1+s}, s \leq 0.3$.

\section{4. Обсуждение результатов}

\section{1. Характерные времена флуктуаций заселенности глубоких центров и частота перескоков в ОПЗ с прыжковой проводимостью}

При прямом смещении светодиодных $p-n$-структур с квантовой ямой $\mathrm{InGaN} / \mathrm{GaN}$, имеющих низкое пороговое напряжение детектирования излучения $V_{\mathrm{th}}=2.2 \mathrm{~B}$, носители заряда инжектируются в активную область, тунне- лируя под барьером, а надбарьерный ток пренебрежимо мал (см., например, $[15,16])$. Глубокий экспоненциальный хвост локализованных состояний в запрещенной зоне $\mathrm{GaN}$, формируемый гауссовыми полосами дефектов типа глубокий донор-акцептор, ответственных за полосы фотолюминесценции (см. обзор [20]), облегчает туннельную инжекцию носителей в квантовую яму [15].

В светодиодах A-C на границе с квантовой ямой имеется слабо легированная область $n$ - GaN $[19,21]$, так что полный ток контролируется электронным туннельным током. Для иллюстрации механизма туннелирования с участием дефектов (defect-assisted tunneling, hopping) будем обращаться к электронному току (см. вставку к рис. 1,a), для простоты пренебрегая при $V_{j}>V_{\text {th }}$ падением напряжения на $p$-области пространственного заряда. Учитывая, что в $\mathrm{GaN}$ хвост состояний зоны проводимости много шире хвоста состояний валентной зоны, а также учитывая, что эффективная масса электронов много меньше эффективной массы дырок, такое приближение может быть справедливым и при ином дизайне структуры светодиода.

Механизм туннельной инжекции через область пространственного заряда в квантовую яму иллюстрируется на вставке к рис. 1, $a$. Электроны с энергиями $E_{0}>E>\mathrm{F}_{e} \quad\left(\mathrm{~F}_{e}-\right.$ электронный квазиуровень Ферми, $E_{0}$ - энергия свободного электрона на границе с квантовой ямой) свободны и движутся с тепловой скоростью в области шириной $w_{\text {th }}\left(E_{t e}\right)$; в области шириной $w_{\text {hop }}\left(E_{t e}\right)$ электроны движутся сквозь $n$-барьер перескоками между состояниями дефектов на транспортных уровнях $E_{t e}=E$. На вставке к рис. $1, a$ схематично показано протекание компоненты тока туннельной инжекции $J_{\mathrm{n}} \rightarrow \mathrm{QW}$ для электронов с энергией $E_{\text {eff, }}$ термоактивированных в нейтральной $n$-области на транспортный уровень $E_{t e}=E_{\text {eff }}$ и формирующих пик в спектре излучения.

Прыжковая проводимость через ОПЗ позволяет снизить инжекционный барьер, но в то же время вносит последовательное прыжковое сопротивление, ограничивающее поток электронов из нейтральной $n$-области. Ограничение тока последовательным прыжковым сопротивлением приводит к кардинальному изменению распределения электрического поля в ОПЗ при прямых смещениях [16]. Полное падение напряжения $V_{j}$ складывается из падения напряжения на $n$-барьере, на области шириной $w_{\text {th }}\left(E_{t e}\right)$, и падения напряжения на внутренней базе $n$-барьера, области $w_{\text {hop }}\left(E_{t e}\right)$. Основной поток электронов протекает на квазиуровне Ферми. По мере приближения электрона к квантовой яме плотность изоэнергетических локализованных состояний на квазиуровне Ферми $N_{t}\left(F_{e}, x\right)$ убывает, вероятность прыжка уменьшается и локальное прыжковое сопротивление $r_{\text {hop }}\left(F_{e}, x\right)$ экспоненциально растет, так что полное прыжковое сопротивление ОПЗ определяется в основном плотностью локализованных состояний у границы с квантовой ямой, $N_{t}\left(F_{e}, 0\right)$. В стационарном состоянии распределение приложенного прямого 
напряжения в ОПЗ определяется распределенным сопротивлением $r_{\text {hop }}\left(F_{e}, x\right)$, и основная часть прямого напряжения падает вблизи квантовой ямы. По мере увеличения прямого напряжения это приводит сначала к постепенному уменьшению электрического поля у квантовой ямы, а затем направление электрического поля вблизи квантовой ямы изменяется [16] (см. вставку к рис. $1, a)$, подобно характеру изменения направления и последующего увеличения электрического поля в базе $p-n$-перехода или в $i$-слое $p-i-n$-структуры при высоких уровнях инжекции. В результате при увеличении прямого смещения $V_{j}$ вершина барьера удаляется от границы, а высота барьера уменьшается мало. Это приводит к ограничению сквозного тока на транспортных уровнях $E_{t e} \geq F_{e}$ более глубокими центрами с большим временем перескока $\tau_{\text {hop }}(E, x)$, вызывая увеличение туннельного потока дырок из квантовой ямы с участием акцепторных состояний дефектов, что сначала ограничивает рост эффективности, а затем вызывает падение эффективности [16].

В рамках этой модели, предложенной и обоснованной в работах $[15,16]$, ток и эффективность должны демонстрировать тенденцию к росту при увеличении плотности состояний на границе с активной областью, пересекаемых квазиуровнем Ферми по мере увеличения прямого смещения. В работах $[15,16]$ показано, что ступенчатые изменения тока и эффективности в светодиодных структурах на $\mathrm{GaN}$ наблюдаются, когда разность квазиуровней Ферми $\Delta F=q V_{j}$ достигает оптических пороговых энергий в спектрах поглощения кристаллов $\mathrm{GaN}$, равных пиковым энергиям красной $h v_{\mathrm{RL}}=1.85$ эВ, желтой $h v_{\mathrm{YL}}=2.2$ эВ, зеленой $h v_{\mathrm{GL}}=2.5$ эВ, голубой $h v_{\mathrm{BL}}=2.85$ эВ и УФ $h v_{\mathrm{UVL}}=3.2$ эВ полос дефектной фотолюминесценции и порогу близкраевой фотолюминесценции $h v_{\mathrm{NBL}} \approx 3.4$ эB $[15,16]$. В работах $[15,16]$ показано, что основными характерными чертами энергетического спектра дефектов в $\mathrm{GaN}$, определяющими вид зависимостей $I\left(V_{j}\right)$ в светодиодных структурах, является резкий рост их плотности в области пороговых энергий 2.2 и 2.85 эB, обусловленный YL- и BL-центрами, ответственными за желтую и голубую фотолюминесценции, а также экспоненциальный хвост плотности состояний вблизи краев свободных зон при $q V_{j}>3.2$ эВ, обусловленный UVL- и NBL-центрами, ответственными за УФ и близкраевую фотолюминесценции. В рамках этой модели можно связать резкий рост тока в диодах $\mathrm{A}$, $\mathrm{B}, \mathrm{C}$ вблизи пороговых напряжений $q V_{j}=2.2$ эВ с YL-центрами и резкий рост тока в области рабочих смещений (см. вставку к рис. 2, $b$ ), наблюдающийся в диоде А при $q V_{j}=3.4 э \mathrm{~B}$, а в диодах В и С при $q V_{j}=2.85$ и 3.2 эВ с NBL-, BL- и UVL-центрами соответственно [16].

Полученные результаты позволяют предположить, что токовый шум в исследованных $p-n$-структурах с $\mathrm{InGaN} / \mathrm{GaN}$-квантовой ямой связан с механизмом прохождения тока и флуктуациями прыжкового сопротивления $n$-области пространственного заряда. Сильный низ- кочастотный токовый шум $1 / f$ при прыжковой проводимости в полупроводниках связывается со случайным распределением примесей в пространстве и экспоненциальной зависимостью частоты прыжков и локальной проводимости от расстояния между центрами, приводящими к флуктуациям занятости центров и флуктуациям сопротивления [22,23].

В $p$-n-структурах с квантовой ямой $\mathrm{InGaN} / \mathrm{GaN}$ с экспоненциально широким распределением плотности состояний в запрещенной зоне $\mathrm{GaN}$ среднее время перескока между центрами при приближении электрона к границе с квантовой ямой экспоненциально увеличивается. Постоянная времени, характеризующая релаксацию заселенности центров на транспортном уровне при его отклонении от стационарного неравновесного заполнения, определяется при прыжковой проводимости распределением локальных времен максвелловской релаксации $\tau_{\mathrm{M}, \mathrm{hop}}\left(E_{t e}, x\right)=\varepsilon_{0} \varepsilon / \varepsilon_{\mathrm{hop}}\left(E_{t e}, x\right)$ [24]. Здесь $\varepsilon_{0}$ и $\varepsilon$ - электрическая постоянная и относительная диэлектрическая проницаемость соответственно.

При движении электрона к квантовой яме прыжками между состояниями на квазиуровне Ферми скорость, с которой он совершает прыжки вблизи нейтральной области, перескакивая между мелкими состояниями с малой энергией локализации и высокой плотностью, намного больше, чем скорость, с которой он „выпрыгивает“ из глубоких состояний вблизи квантовой ямы, с большей энергией локализации и меньшей плотностью. Это позволяет условно разделить состояния на „быстрые“ и „медленные“ на основе их энергии локализации. При увеличении смещения $V_{j}$ и перемещении квазиуровня Ферми вверх, область с высокой проводимостью $\sigma_{\text {hор }}$ и „быстрыми“ состояниями приближается к квантовой яме, а область с низкой проводимостью $\sigma_{\text {hор }}$ и „медленными“ состояниями, сужается. Увеличение смещения на $\Delta V_{j}$ приводит к захвату и накоплению электронов на „медленных“ состояниях, что проявляется в затухании переходного электронного тока, но постепенное их заполнение и соответстветствующее перемещение квазиуровня Ферми вверх на $\Delta F_{e}=\Delta V_{j}$ приводит к инерционному увеличению проводимости $\sigma_{\text {hор }}$ и увеличению переходного тока до стационарного значения [15].

Как показали измерения переходных токов, при допороговых напряжениях светодиоды на основе $\mathrm{GaN}$ демонстрируют емкостной динамический отклик: переходной ток на несколько порядков превышает стационарный и затухает со временем до величины стационарного тока как $I \sim 1 / t$, при этом временной масштаб $\tau_{\text {hор увели- }}$ чивается от наносекундного до миллисекундного (см. работу [24] и ссылки в ней). С увеличением смещения характер динамического отклика меняется на индуктивный: наблюдается отрицательная дифференциальная емкость, а переходной ток медленно увеличивается со временем до стационарной величины $[16,24]$. В диодах А, В и С измеряемая на частоте 1 МГц емкость падает до нуля при пиковых напряжениях $V_{j \mathrm{p}}=2,7,2.8$ и $2.93 \mathrm{~B}$, 
а при $V_{j}>V_{j \mathrm{p}}$ наблюдаются отрицательная дифференциальная емкость и падение эффективности (см. рис. $1, b$ в работе [16]). При индуктивном характере динамического отклика диода $(C<0)$ характерное время установления распределения напряжения в ОПЗ определяется, как в параллельной RL-цепи, величиной эквивалентной индуктивности, равной $L=-1 /\left(4 \pi^{2} f^{2} C\right)$, а постоянная времени, характеризующая релаксацию, равна $\tau_{\mathrm{RL}}=L / R$, где $R$ - прыжковое сопротивление области, примыкающей к границе с квантовой ямой.

В этой модели экранирование флуктуаций заселенности глубоких центров у границы с квантовой ямой, определяющих величину полного прыжкового сопротивления ОПЗ, вызывает модуляцию проводимости ОПЗ. Экранирование флуктуации заселенности происходит за счет прыжкового тока электронов в область, примыкающую к границе с квантовой ямой (или, в зависимости от знака флуктуации, из этой области), что приводит к инерционному увеличению (или уменьшению) ее проводимости. Флуктуации заселенности центров экспоненциально затухают во времени с постоянной времени $\tau_{\mathrm{RL}}$, что приводит к частотной зависимости плотности токового шума, определяемой лоренцовской функцией [22]:

$$
S_{I} \sim \frac{\tau_{\mathrm{RL}}}{1+4 \pi^{2} f^{2} \tau_{\mathrm{RL}}^{2}} .
$$

В этом случае плотность токового шума в области низких частот $2 \pi f \tau_{\mathrm{RL}} \ll 1$ не зависит от частоты анализа, а в области высоких частот $2 \pi f \tau_{\mathrm{RL}} \gg 1$ уменьшается с ростом частоты как $S_{I} \sim 1 / f^{2}$.

В рамках этой модели особенности частотных спектров $S_{I}(f)$ (рис. 3), измеренных при рабочем токе $I=20$ мА: слабая зависимость плотности шума диода А от частоты (кривая 1) в области низких частот $2 \pi f \tau_{\mathrm{RL}} \ll 1$ и сверхлинейный спад в области высоких частот $2 \pi f \tau_{\mathrm{RL}} \gg 1$, а также сверхлинейный спад с частотой в диоде $\mathrm{C}$ (кривая 3 ) и спад, близкий в области низких частот к $S_{I} \sim 1 / f^{2}$ в диоде В (кривая 2 ), могут быть связаны с преимущественным вкладом в плотность шума флуктуаций занятости центров вблизи границы с квантовой ямой. Низкочастотный сдвиг сверхлинейного спада плотности шума в диодах В и С, наиболее сильный в диоде В, объясняется, согласно (1), значительно меньшей по сравнению с диодом А величиной их дифференциального сопротивления при $V_{j}>V_{j \mathrm{p}}$, наименьшей в диоде В.

\section{2. Прыжковый транспорт в ОПЗ как источник избыточного низкочастотного шума}

Как можно видеть из токовых зависимостей плотности шума $S_{I}(I)$ диодов $\mathrm{A}-\mathrm{C}$, измеренных на различных частотах анализа (рис. $1, a-c$, кривые $1-4$ ), отмеченные в разд. 4.1 особенности частотных зависимостей плотности токового шума $S_{I}(f)$ : появление ступеней и сверхлинейный спадов на кривых $S_{I}(f)$ характерны для токов, близких к рабочим, при которых эффективность падает с ростом тока наиболее сильно (кривые 5), а фактор идеальности быстро уменьшается (рис. 2, $a-c$, кривые 5,6). В то же время при уменьшении тока до пикового тока $I_{\mathrm{p}}$, характер спада плотности шума с увеличением частоты анализа становится более близким к линейному: $S_{I}(f) \sim 1 / f$.

Мы связываем это изменение частотного спектра шума с доминированием активного динамического отклика диода на приращение напряжения в области $V_{j \mathrm{p}}>V_{j}>2.5 \mathrm{~B}$, в которой емкостной спад переходного тока компенсируется инерционным ростом тока. В диодах А, В и С активная проводимость на частоте $f=1$ Мгц, $G_{\text {ac }}(f)$, превышающая проводимость на постоянном токе, $G_{\mathrm{dc}}$, при $V_{j}<2.5 \mathrm{~B}$ становится равной $G_{\mathrm{dc}}$, причем у всех диодов равенство проводимостей $G_{\mathrm{ac}}(f)=G_{\mathrm{dc}}$ наблюдается при одном и том же смещении $q V_{j}=2.5$ эВ [16]. Это позволяет считать, что при напряжениях, ненамного превышающих $q V_{j}=2.5$ эВ, флуктуации занятости центров у границы с квантовой ямой должны приводить к флуктуациям проводимости, характерным для сопротивления.

В однородном материале спектральная плотность токового шума $1 / f S_{I}(I)$ уменьшается с увеличением среднего числа электронов, принимающих участие в проводимости $\bar{N} \neq f(I)$, и растет квадратично с ростом тока [25]:

$$
S_{I}=\frac{\alpha}{f \bar{N}} I^{2},
$$

где $\alpha$ - параметр Хоуге. В идеальном $p-n$-переходе $\bar{N} \sim 1 / r_{d}\left(r_{d}=k T / q I-\right.$ дифференциальное сопротивление $p-n$-перехода) и линейно растет с током, что приводит, согласно (2), к линейному росту плотности шума: $S_{I}(I) \sim I$. В $p-n$-структуре с прыжковой проводимостью через ОПЗ $r_{d}=d V_{j} / d I=n_{j}(I) k T / q I$ и плотность шума должна увеличиваться при больших величинах $n_{j}(I)$, a ее токовая зависимость $S_{I}(I)$ должна приближаться к квадратичной, характерной для сопротивления, $S_{I}(I) \sim I^{2}$, когда $n_{j}(I)$ увеличивается с ростом тока; к линейной, характерной для $p-n$-перехода, в случае $n_{j}(I)=\mathrm{const}$ и к сублинейной, когда $n_{j}(I)$ уменьшается с ростом тока [13]. Эти модельные соображения позволяют связать высокий уровень шума и его сверхлинейный рост, близкий к $S_{I} \propto I^{2}$, вблизи пикового тока $I_{\mathrm{p}}>I>I_{\mathrm{th}}$ в диоде А (рис. 2, $a$, кривые 1-4), а также сильный шум в диоде В в области малых токов $I_{\mathrm{p}}>I>I_{\text {th }}$ (рис. 2, $b$, кривые 1-4) с большой величиной фактора идеальности и характером зависимостей $n_{j}(I)$ в этих диодах (кривые 6). В диодах А и С в области малых токов $I_{\mathrm{p}}>I>I_{\mathrm{th}}$, где эффективность растет с током, наблюдается низкий уровень шума. Рост плотности токового шума и ограничение роста эффективности в области напряжений $V_{j} \approx V_{j \text { p }}$ можно связать с недостаточно высокой плотностью YL-центров, особенно низкой в зеленых светодиодах, для которых характерна малая крутизна ВАХ (большая величина фактора идеальности $\mathrm{BAX)} \mathrm{в} \mathrm{этой} \mathrm{области} \mathrm{напряжений} \mathrm{[16].}$ 


\section{5. Заключение}

Измерения токовых зависимостей спектральной плотности токового шума и квантовой эффективности в „зеленых“ и „синих“ GaN-светодиодах показали, что падение эффективности с ростом тока сопровождается сильным ростом уровня шума. Основным источником избыточного низкочастотного шума является туннелирование электронов с участием дефектов через $n$-барьер квантовой ямы InGaN/GaN. Прыжковое сопротивление ОПЗ определяется энергетическим спектром дефектов у границы с квантовой ямой, состоящим из гауссовых полос состояний центров окраски в $\mathrm{GaN}$. С увеличением уровня инжекции последовательное „прыжковое“ сопротивление ОПЗ с высоким уровнем токового шума все больше ограничивает поток термоактивированных электронов в квантовую яму. При достаточно большом токе падение напряжения на прыжковом сопротивлении ОПЗ приводит к изменению направления поля вблизи квантовой ямы. В результате увеличения туннельного потока дырок из квантовой ямы эффективность излучения падает. Усиление влияния прыжкового сопротивления на сквозной ток приводит также к уменьшению демпфирования шума прыжкового сопротивления и росту уровня токового шума светодиода. Частотный спектр плотности токового шума определяется частотой перескоков и временем максвелловской релаксации у границы с квантовой ямой и имеет вид лоренцовского спектра.

\section{Список литературы}

[1] Z. Yatabe, J.T. Asubar, T. Hashizume. J. Phys. D: Appl. Phys., 49, 393001 (2016).

[2] M.E. Levinshtein, S.L. Rumyantsev, R. Gaska, J.W. Yang, M.S. Shur. Appl. Phys. Lett., 73, 1089 (1998).

[3] S. Bychikhin, D. Pogany, L.K.J. Vandamme, G. Meneghesso, E. Zanoni. J. Appl. Phys., 97, 123714 (2005).

[4] K.K. Leung, W.K. Fong, P.K.L. Chan, C. Surya. J. Appl. Phys., 107, 073103 (2010).

[5] D. Ursutiu, B.K. Jones. Semicond. Sci. Technol., 11, 1133 (1996).

[6] V. Palenskis, J. Matukas, S. Pralgauskaite. Sol. St. Electron., 54, 781 (2010).

[7] Z.L. Li, S. Tripathy, P.T. Lai, H.W. Choi. J. Appl. Phys., 106, 094507 (2009).

[8] M.E. Levinshtein, F. Pascal, S. Contreras, W. Knap, S.L. Rumyantsev, R. Gaska, J.W. Yang, M.S. Shur. Appl. Phys. Lett., 72, 3053 (1998).

[9] Н.В. Дьяконова, М.Е. Левинштейн, С.Л. Румянцев. ФТП, 25 (12), 2065 (1991).

[10] A.P. Dmitriev, M.E. Levinshtein, S.L. Rumyantsev, M.S. Shur. J. Appl. Phys., 97, 123706 (2005).

[11] J.A. Garrido, B.E. Foutz, J.A. Smart, J.R. Shealy, M.J. Murphy, W.J. Schaff, L.F. Eastman, E. Muñoz. Appl. Phys. Lett., 76, 3442 (2000).

[12] S. Sawyer, S.L. Rumyantsev, M.S. Shur, N. Pala, Yu. Bilenko, J.P. Zhang, X. Hu, A. Lunev, J. Deng, R. Gaska. J. Appl. Phys., 100, 034504 (2006).
[13] Н.И. Бочкарева, А.М. Иванов, А.В. Клочков, В.С. Коготков, Ю.Т. Ребане, М.В. Вирко, Ю.Г. Шретер. ФТП, 49 (6), 847 (2015).

[14] Н.И. Бочкарева, А.М. Иванов, А.В. Клочков, В.А. Тарала, Ю.Г. Шретер. Письма ЖТФ, 42 (22), 1 (2016).

[15] Н.И. Бочкарева, И.А. Шеремет, Ю.Г. Шретер. ФТП, 50 , 1387 (2016).

[16] Н.И. Бочкарева, Ю.Г. Шретер. ФТП, 52, 796 (2018).

[17] D. Schiavon, M. Binder, M. Peter, B. Galler, P. Drechsel, F. Scholz. Phys. Status Solidi B, 250 (2), 283 (2013).

[18] S. Nakamura, M. Senoh, N. Iwasa, S. Nagahama, T. Yamada, T. Mukai. Jpn. J. Appl. Phys., 34, L1332 (1995).

[19] T. Mukai, K. Takekava, S. Nakamura. Jpn. J. Appl. Phys., 37, L839 (1996).

[20] M.A. Reshchikov, H. Morkoç. J. Appl. Phys., 97, 061301 (2005).

[21] Н.И. Бочкарева, В.В. Вороненков, Р.И. Горбунов, Ф.Е. Латышев, Ю.С. Леликов, Ю.Т. Ребане, А.И. Цюк, Ю.Г. Шретер. ФТП, 47, 115 (2013).

[22] Ш.М. Коган, Б.И. Шкловский. ФТП, 15, 1049 (1981).

[23] B.I. Shklovskii. Phys. Rev. B, 67, 045201 (2003).

[24] Н.И. Бочкарева, В.В. Вороненков, Р.И. Горбунов, М.В. Вирко, В.С. Коготков, А.А. Леонидов, П.Н. ВоронцовВельяминов, И.А. Шеремет, Ю.Г. Шретер. ФТП, 51 (9), 1235 (2017).

[25] F.N. Hooge. Physica, 60, 130 (1972).

Редактор А.Н. Смирнов

\section{Current noise and efficiency droop of light-emitting diodes due to defect-related tunneling of carriers from InGaN/GaN quantum well}

\author{
N.I. Bochkareva, A.M. Ivanov, A.V. Klochkov, \\ Y.G. Shreter \\ loffe Institute, \\ 194021 St. Petersburg, Russia
}

\begin{abstract}
Measurements of current dependencies of the noise spectral density and quantum efficiency in the green and blue LEDs with InGaN/GaN quantum wells were carried out. It is shown, that a noise level strongly increases at large currents in which there is a quantum efficiency droop. A mechanism of current noise formation is associated with a hopping transport via deep states of the color centers in GaN through the spacecharge region of InGaN/GaN quantum well $n$-barrier. The source of the noise is the hopping resistance, which limits the current of thermally activated electrons in the quantum well. The efficiency droop and increase in noise level is associated with a change in the direction of the electric field near the quantum well at high injection levels and an increase in tunnel leakage of holes from the quantum well. It is shown that the experimental frequency noise spectra, which have the shape of Lorentz spectrum at operating currents, are related to the frequency of hopping between deep centers near the InGaN/GaN quantum well and Maxwell relaxation in the space-charge region.
\end{abstract}

